\title{
Examining the effects of social media in co-located classrooms: A case study based on SpeakUp
}

\author{
María Jesús Rodríguez-Triana, Adrian Holzer, Luis P. Prieto, and Denis Gillet \\ École Polytechnique Fédérale de Lausanne, Switzerland \\ \{maria.rodrigueztriana, adrian.holzer, luis.prieto, denis.gillet\}@epfl.ch
}

\begin{abstract}
The broad availability of mobile computing devices has prompted the apparition of social media applications that support teaching and learning. However, so far, there is conflicting evidence as to whether the benefits such applications provide in terms of engagement and interaction, outweigh their potential cost as a source of distraction. To help in clarifying these issues, the present paper presents a case study on the impact of using SpeakUp (an app aimed at promoting student participation through anonymous chatrooms) in an authentic face-to-face learning scenario. Concretely, we focus on the connection between SpeakUp and the student engagement, distraction, social interaction, and the influence of the teachers' style. Our findings highlight that SpeakUp favored students' engagement and social interaction, but they also point towards its limitations in keeping students communicating about content relevant to the course.
\end{abstract}

Keywords: Social media, engagement, attention, interaction, learning, teaching.

\section{Introduction}

Multiple social media applications are appearing to support teaching and learning, leveraging the broad access to mobile devices (e.g., in "bring your own device" approaches). However, there is conflicting evidence on whether the use of mobile technologies in the classroom is positive (e.g., improving student participation) [33] or negative (e.g. distracting students due to multitasking) $[28,35]$.

In this context, we are interested in studying how to use social media effectively in the classroom. This paper focuses on the pedagogical use of SpeakUp, a mobile app aimed to promote student participation in face-to-face sessions. In SpeakUp, students can anonymously join chatrooms, post messages and vote on them.

Since the mere introduction of social media in educational contexts does not ensure a positive effect, this paper analyses the impact of SpeakUp in an authentic learning scenario carried out with first-year (bachelor) university students. In particular, this paper explores the following research question: does SpeakUp favor situations that lead to learning? To answer this question, we structured the study according to the following topics: active participation [25] (i.e., engagement), attention [17] (i.e., remaining on-task), and social interaction [6] (on relevant content).

The CSCL-EREM framework [19] guided the formalization of this case study, as well as the data gathering and analyses, leading us to use multiple informants (students, teachers, researchers and the technology used), different data gathering techniques (observations, questionnaires, SpeakUp logs, and user comments in the app), and mixed 
methods analyses, including: student attendance to the session, teacher and student participation face-to-face and via SpeakUp, content of the comments, as well as teacher and student perceptions about the impact on engagement, attention and interaction.

The paper is structured as follows: Section 2 reviews previous research on the usage of social media for educational purposes; Section 3 introduces the SpeakUp app and its main functionalities; Section 4 describes the research methodology followed in the present case study, while Section 5 details the main results of the data analyses and that are later discussed in Section 6 together with the main conclusions and the future work.

\section{Related work}

Historical overview. Social interaction in the classroom is considered by numerous researchers as a conditio sine qua non for learning $[8,21]$. Providing learners with a digital channel for interaction can be traced back to the 80 's when IBM started to experiment with student interaction systems [16]. Many of these systems are based on reactive interaction where teachers can conduct live polling by asking multiple choice questions and students answer by pressing a button on a clicker. Studies on clickers show that they can foster more participation in the classroom, and that students generally have a positive attitude towards them (e.g., $[3,9,32,34])$. On top of the reactive channel, some systems provide a proactive channel, where students can post questions and comments. With the rise of mobile devices, systems also started relying on the students' own devices. An early effort in this direction was the TXT-2-LRN [29] mobile system, with which students could send free-form SMSs to the teachers.

Students' perceptions. More recently, systems also include a social media layer, where students can vote and comment on each other's contributions (e.g., ClassCommons [7], Fragmented Social Mirror [2], Pigeonhole Live [11], Backchan.nl [13], or SpeakUp [14, 15]). Mainstream social media, such as Twitter [26, 27] and Reddit, are also popular when attempting to foster interaction between speakers and their audience in both conferences and classrooms. Research investigating the use of such social media applications in the classroom generally concludes that students perceive such systems as positive and that they feel it increases interactivity [1,2,9,13-15,29]. Furthermore, students often prefer to use a digital channel to interact instead of raising their hand [29].

Teachers' opinions. The Pearson education service company conducted a survey with 7969 U.S. higher education teachers to better understand the bigger picture of the social media usage by teachers [30]. The survey finds that teachers are generally aware of social media and they are using it in their private lives $(70.3 \%$ of faculty use it at least once per month). The use of social media in the classroom lags behind the usage in their personal lives $(41.0 \%)$ but is increasing every year. Teachers see social media and technology as having a "considerable potential" for learning. However, $56.0 \%$ of teachers also consider that social media in class can be more distracting than helpful.

Potential shortcomings. The issue of distraction and multitasking in education is receiving increased attention, with conflicting results so far. Certain research suggests that laptop multitasking hinders learning for both users and nearby peers [28], and that providing slides to students can affect performance adversely [22,35]. On the other hand, researchers also argue that it is possible to take advantage of social media in the 
classroom by embracing multitasking, which students seem to able to effectively do in the classroom $[20,36]$. A recent meta-analysis on the use of mobile devices in the classroom nuances these claims and shows a moderate positive learning effects [33].

This paper aims at better understanding whether, and under what circumstances, social media usage in the classroom may have a positive impact.

\section{SpeakUp}

SpeakUp is a social media app designed to foster participation in co-located situations where such interaction is difficult, either within the audience or between the speaker and the audience (e.g., a university lecture with a large number of students, or a conference). In a typical usage scenario with SpeakUp, teachers create a chatroom that students can join by typing its number as shown in Figure 1.1. Note that any user can join such rooms without login or registration (enabling an immediate use of the app).
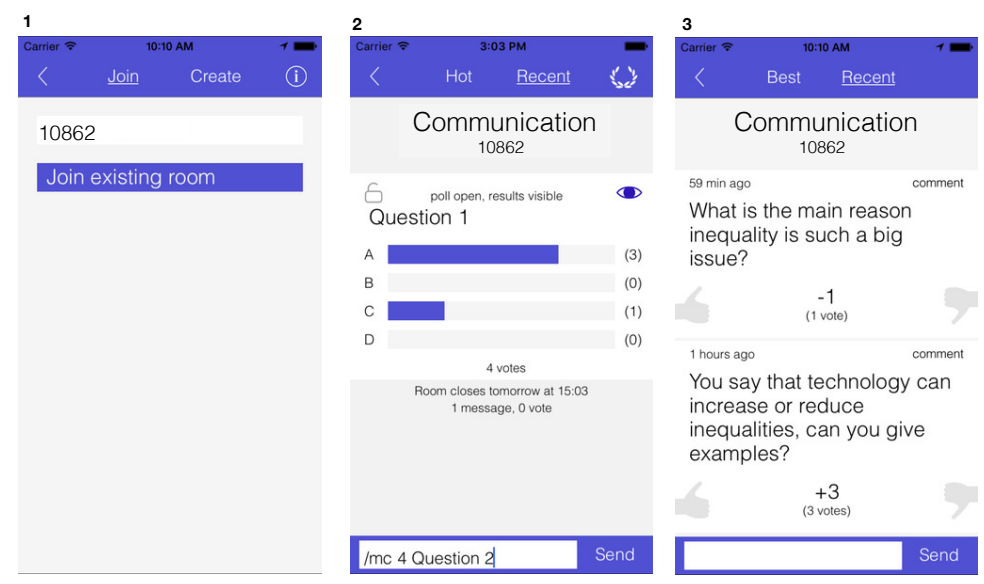

Fig. 1: Screenshots of the SpeakUp mobile app. (1) joining a chatroom. (2) creating a multiple choice question in the chatroom. (3) viewing messages in the chatroom ordered by time or score.

Inside the chatroom, any user can post text messages, comment on existing messages, and vote them (up or down, see Figure 1.3). Each message has a score, which shows the difference between the number of upvotes and downvotes. For instance, the top message in Figure 1.3 has a score of -1 and the bottom message a score of +3 . The chatroom creator, i.e., the teacher, can create multiple choice messages (Figure 1.2) for students to answer. Inside the chatroom messages are sorted either by time or by score.

Furthermore, in the chatroom all users are anonymous, thus fostering the expression of more uninhibited points of view. This implies that users interact, not directly with one another, but rather on the basis of the content posted by the different anonymous users.

Classroom interaction in a lesson using SpeakUp can occur along the face-to-face (f2f) channel (i.e., teachers and students interacting orally), as well as along a digital channel (i.e., posting comments and voting on SpeakUp). There can also be transitions from one channel to the other. For instance, a teacher can instruct students to answer a 
poll on SpeakUp, or explicitly ask students to post messages on SpeakUp. Conversely, questions posed by students on SpeakUp, can be answered by teachers orally.

\section{Methodology}

The present study is framed within a wider research effort whose general goal is to understand how social media can be used effectively in the classroom. Towards this aim, several exploratory studies have been performed in the past on the use of SpeakUp in classrooms $[14,15]$, in which SpeakUp was deemed easy to use, and motivating for students to participate more in lectures. In turn, the present study is the first of a series in which we aim to evaluate the effectiveness of SpeakUp to foster learning in more details using authentic educational settings [5]. We also aim to assess its potential role in the distraction of students, and its relations with various teaching strategies and styles.

This concern with deep evaluation of a social learning tool has led us to use a case study methodology [31], structured using the Computer Supported Collaborative Learning - Evaluand Oriented Responsive Evaluation Model (CSCL-EREM, see [19]) framework. This framework was designed specifically to evaluate the impact of TEL interventions, especially in authentic settings. Hereafter, we discuss the research issue and topic, the data sources, and the data analyses (see Figure 2).
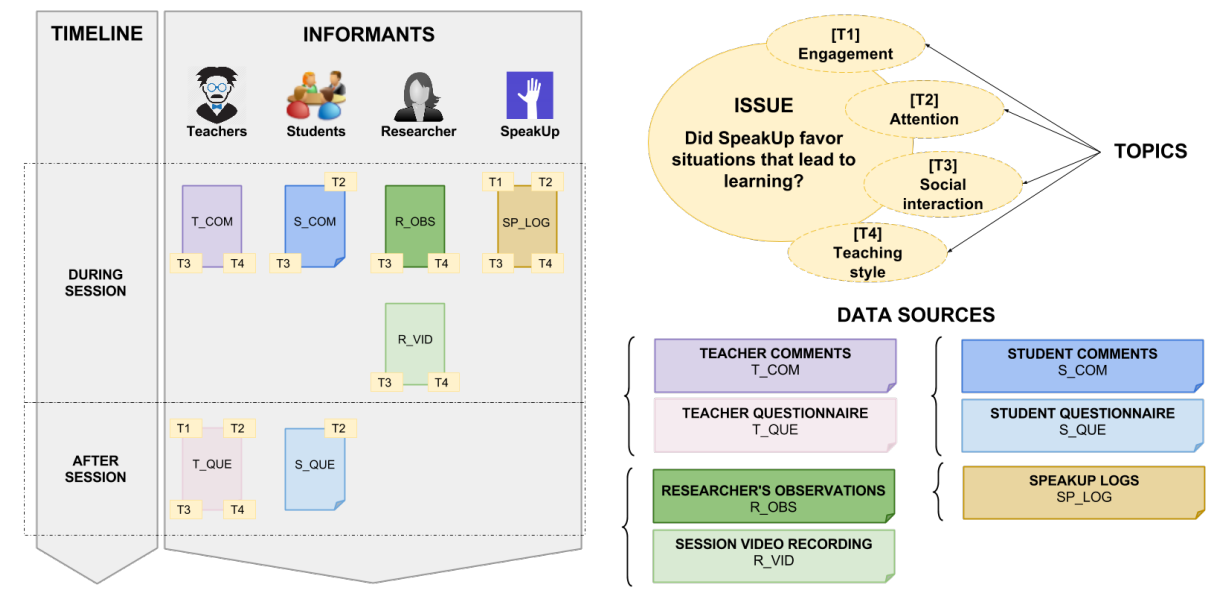

Fig. 2: Diagram representing the issues, topics, data sources and informants used in the case study

Research issue and topics. Guided by this framework, we organised the perspective of the case study around the definition of an issue. An issue can be understood as a troubling choice, a tension, an organizational perplexity or a problem. In this case study, the main issue is defined as: does SpeakUp favor situations that lead to learning, such as active participation (i.e., engagement), attention (i.e., focus on-task), and social interaction (on relevant content)? Then, following an anticipated data reduction procedure (common in qualitative data analysis [24]), this issue is illuminated by answering a number of informative questions, clustered around four topics (see Figure 2). These 
topics are related to the users' active participation and engagement with the tool (T1), its effects on attention (i.e., focusing vs. distracting from the lesson topic, T2), and on the classroom social interactions (T3). Finally, another topic explores the interactions of teacher actions and style with the different kinds of SpeakUp usage (T4).

Data gathering, informants and data sources. We use a mixed method approach [10, 4] combining quantitative and qualitative data coming from four types of informants (two teachers, 145 students, one observer, plus the SpeakUp logs) using different data gathering techniques: questionnaires, logfile analysis, observations, video recordings, and student contributions in SpeakUp. This mixed methods approach is commonly used in TEL research [18] and promoted by the CSCL-EREM methodology in order to obtain different perspectives about the evaluand (the object of the evaluation, in our case the use of SpeakUp in the lesson), thus enriching the evaluation process.

Data analysis. Different quantitative (descriptive statistics and exploratory computational analyses) and qualitative analyses (manual coding of the messages generated by the users, see below) have been performed on the data. Then, the results from these analyses were triangulated [12] to increase the trustworthiness of our findings.

In order to better understand the aforementioned aspects of engagement, attention and social interaction, we manually coded all the messages and comments generated during the lesson, into two main categories: Messages that are relevant for learning and messages not relevant for learning, similarly to previous studies on SpeakUp [14]. We further divided these main categories in four sub-categories, inspired by those proposed by McCarthy [23]: the relevant message were divided into content related messages (i.e., questions or comment about the content of the course), organisation related messages (i.e, messages related to team and course organisation), SpeakUp related messages (i.e, messages discussing SpeakUp itself) and miscellaneous messages (i.e, messages such as greetings and policing). Non-relevant messages were also divided into content-related (i.e., messages that discuss course content but are not relevant to learning), SpeakUp related (i.e., not relevant messages related to the use of SpeakUp) and miscelleanous messages. We also added a social message category (i.e., non-relevant messages about people) and a bullying message category (i.e., non-relevant messages with negative social connotations). Figure 3 shows examples of messages in each category. Furthermore, each message was also labeled as comment, answer or question, and tags were also added about the direction of the interaction: students to teachers, students to students, students to all, and teachers to students.

In a similar way, and in order to understand these topics as they occurred in the face-to-face channel of the classroom, the video recording of the lesson was also coded, according to the following categories: Which actor was speaking at each moment during the lesson (e.g., each of the three teachers present, or one of the students); what action was being performed at that moment (e.g., presentation/lecturing, asking questions, providing answers, noting technical or other kinds of problems); who was the target of the interaction, if any (e.g., a teacher, students, or all the class); and finally, what supporting resources were being used, if any (e.g., slides, videos, SpeakUp). 


\begin{tabular}{|c|l|c|l|l|}
\hline Relevancy & Category & $\#$ & \multicolumn{3}{c|}{ Examples } \\
\hline \multirow{4}{*}{ Relevant } & Content & 42 & I don't remember anything about probabilities & Bays Formula \\
\cline { 2 - 6 } & SpeakUp & 34 & Are we anonymous here? & This app is ruining my battery \\
\cline { 2 - 6 } & Organisation & 17 & Do they know that there is only 10 min left in the class? & Looking for a group \\
\cline { 2 - 6 } & Misc & 13 & Hello & some respect please! \\
\hline \multirow{5}{*}{$\begin{array}{c}\text { Non } \\
\text { relevant }\end{array}$} & Content & 60 & earth is flat & LOVE CNN \\
\cline { 2 - 6 } & SpeakUp & 17 & If I get 100 likes, I will take off my clothes & Telegram channel : comA ? \\
& Social & 83 & He endorses the socialist party's initiative & Im sure he's French \\
\cline { 2 - 6 } & Misc & 59 & Let's ask Jawed & lam YourMother \\
\cline { 2 - 6 } & Bullying & 9 & homo & answer of a blond \\
\hline
\end{tabular}

Fig. 3: Examples of SpeakUp message categories.

\section{Case Study}

The different quantitative and qualitative sources detailed in Section 4 were analysed and triangulated to illuminate the issue and topics addressed in the case study. This section presents the results obtained after presenting the context in more details.

\subsection{Context}

The case study took place in the first lecture of a Communication course at the École Polytechnique Fédérale de Lausanne in Switzerland. In this, which lasted for 90 minutes, 145 students (38 female) were present. This Communication course, which discusses different kinds of communication channels, social media platforms and technologyenhanced learning, is part of the Global Issues program, which aims at introducing first-year undergraduate engineering students to interdisciplinary topics and soft skills. A particularity of the programme is that each course is taught by an interdisciplinary research team covering engineering and social science expertise. In this communication course, the teaching team was composed of three lecturers with expertise in social media, information systems, behavioral sciences and management.

The lecturers were familiar with the usage of social media in the classroom, as they had already used social media apps such as Twitter or SpeakUp in their practice. To understand the attitude of students towards technology, we conducted a voluntary questionnaire at the beginning of the session (based on 7-point Likert scale questions). The respondents $(\mathrm{N}=140)$ considered that technologies are useful in the classroom (average Likert score $\mu=5.57)$ and there should be more interaction in the courses $(\mu=4.62)$. Many students asserted that they feel quite free to express what they think in class $(\mu=4.60)$, but also that they often have questions that they do not ask $(\mu=4.52)$. Furthermore, students had a variety of opinions on whether anonymity could be important in order to express what they think during the courses $(\mu=4.09)$.

During this course, SpeakUp was introduced as a communication channel with students to increase interaction, but it also had another pedagogical purpose: since the course deals with communication channels, social media and TEL, SpeakUp would provide students with hands-on experience of many of the subjects studied in class. 


\subsection{Student engagement (Topic 1)}

Teachers, via the [T_QUE] questionnaire, perceived the app as engaging for the students ( $\mu=5$ in a 5-point Likert scale). The teachers pointed out that the main aspects triggering the high engagement could be the possibility of getting responses quickly without being exposed to the whole audience, the anonymity, the potential to know and react on what others think, as well as the opportunity to interact with everyone.

As an overview, if we compare the number of students attending the session (145) [R_OBS] with those joining the SpeakUp chatroom (147) [SP_LOG], we may infer that almost everyone used the tool, even though such use was not compulsory. The number of students registered in SpeakUp was higher than the actual students participating in the face-to-face session, due to the fact that some students started using the app from their phone, and then switched to using it from their computer [R_OBS].

Figure 4 shows how much teachers and students participated face-to-face and via SpeakUp throughout the session (from 16:15 to 18:00). Face-to-face activity is measured in minutes of active participation extracted from the video [R_VID]. Concretely, in the face-to-face channel, teachers were speaking for about 77 minutes and students 11 minutes. In the case of SpeakUp, the participation is measured according to the number of actions [SP_LOG], obtaining a total of 51 and 3841 actions carried out by teachers and students respectively. Looking at Figure 4 we can identify a certain connection with the events happening face-to-face [R_OBS, R_VID]. For example, although the teachers used SpeakUp from the very beginning of the lesson (e.g., adding welcome messages), the app was presented to the students around 16:35, reason why the students started using it later. Then, there was a break of 15 minutes in the session at 17:10, but students continued using the app during this period. Besides, the main peaks of activity correspond to moments in which teachers asked explicitly to use the app in order to answer a poll (e.g., around 17:15) or to write down some ideas about certain topics.
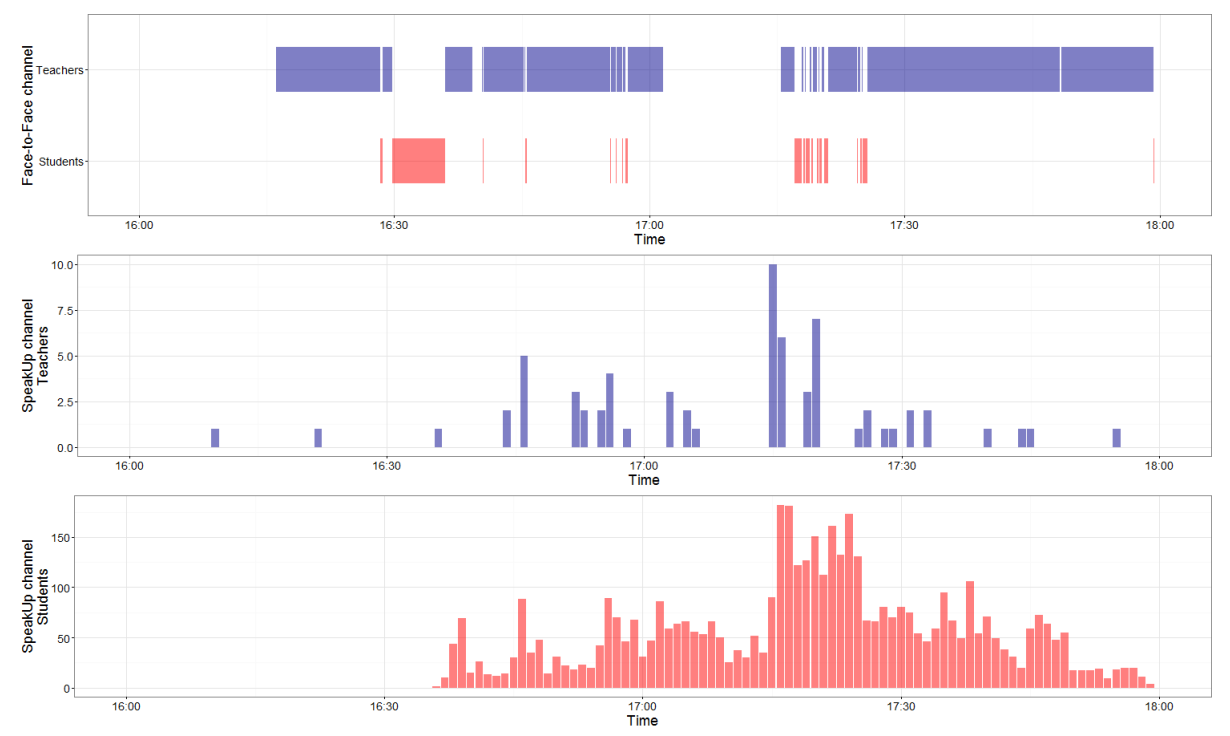

Fig. 4: Face-to-face and SpeakUp-mediated participation during the session. 
Based on the user activity (e.g., number of posted messages, number of likes and dislikes, etc.) [SP_LOG], we have carried out a bottom-up clustering analysis (using a kmeans clustering algorithm with $\mathrm{k}=6$, chosen in terms of within-groups sum of squares), leading to the kinds of users detailed in Table 1. These clusters include large groups of students with low amounts of active usage of SpeakUp (e.g., "Passive"), but also smaller clusters of students with very peculiar engagement patterns (e.g., "Very proactive", which create a large number of messages and votes; or "Super-active voters", who do an unusual amount of voting - especially dislikes -, and very little else).

\begin{tabular}{|c|c|c|c|c|c|c|c|c|}
\hline Clusters & 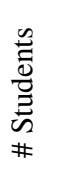 & 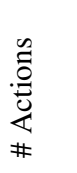 & 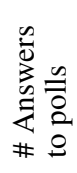 & 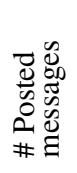 & 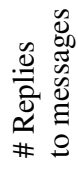 & $\frac{0}{0}$ & $\frac{\mathscr{d}}{\ddot{a}}$ & 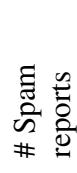 \\
\hline "Passive" & 77 & 7 & 0 & 0 & 0 & 4 & 2 & 0 \\
\hline "Semi-passive" & 36 & 14 & 1 & 1 & 0 & 8 & 5 & 0 \\
\hline "Pro-active/reactive" & 6 & 38 & 1 & 7 & 2 & 17 & 10 & 1 \\
\hline "Mildly pro-active" & 22 & 63 & 1 & 5 & 0 & 32 & 25 & 0 \\
\hline "Very pro-active" & 3 & 143 & 1 & 19 & 1 & 78 & 44 & 0 \\
\hline "Super-active voters" & 4 & 190 & 1 & 0 & 0 & 58 & 130 & 0 \\
\hline
\end{tabular}

Table 1: Types of students based on their interaction with SpeakUp. The action values represent the average values for the cluster.

\subsection{Student attention (Topic 2)}

From the teachers' perspective [T_QUE], SpeakUp had no clear impact on the student attention ( $\mu=3$ in a 5-point Likert scale). They found that although SpeakUp enabled an open channel for topics which might not be related to the course, the app took up one screen of the students' devices, increasing the chances of gathering focused and distracted students. Besides, teachers considered that it might be hard for students to pay attention to both the face-to-face and SpeakUp channels simultaneously.

A minority of students considered that the app did distract them $(18.4 \%, \mathrm{~N}=65$, [S_QUE] see Figure 5, left). However, among student comments [S_COM], only 30.7\% of the messages (out of a total of 322) were categorised as relevant (relevance ratio ${ }^{1}=$ -0.38). These relevant messages were related to the learning content presented during the lesson, the course details, the organisers (i.e., teachers and teacher assistants). It should be noted that the mean scores provided by students - sum of likes and dislikes are slightly higher for relevant $(\tilde{x}=1.16)$ than for non-relevant messages $(\tilde{x}=0.89)$.

To get a picture of the quality of the contribution of each student, Figure 5 (right) depicts the distribution of students in terms of their relevancy ratio. This diagram shows that many of the students sent mainly non-relevant messages, while just 12 students (out of 68 students who generated any kind of message) sent mostly relevant messages; a significant amount of students sent both relevant and not relevant messages.

\footnotetext{
${ }^{1}$ Calculated as: (relevant_posts - non_relevant_posts $) /($ relevant_posts + non_relevant_posts $)$. Hence, ranging from -1 (all messages irrelevant) to +1 (all messages relevant).
} 

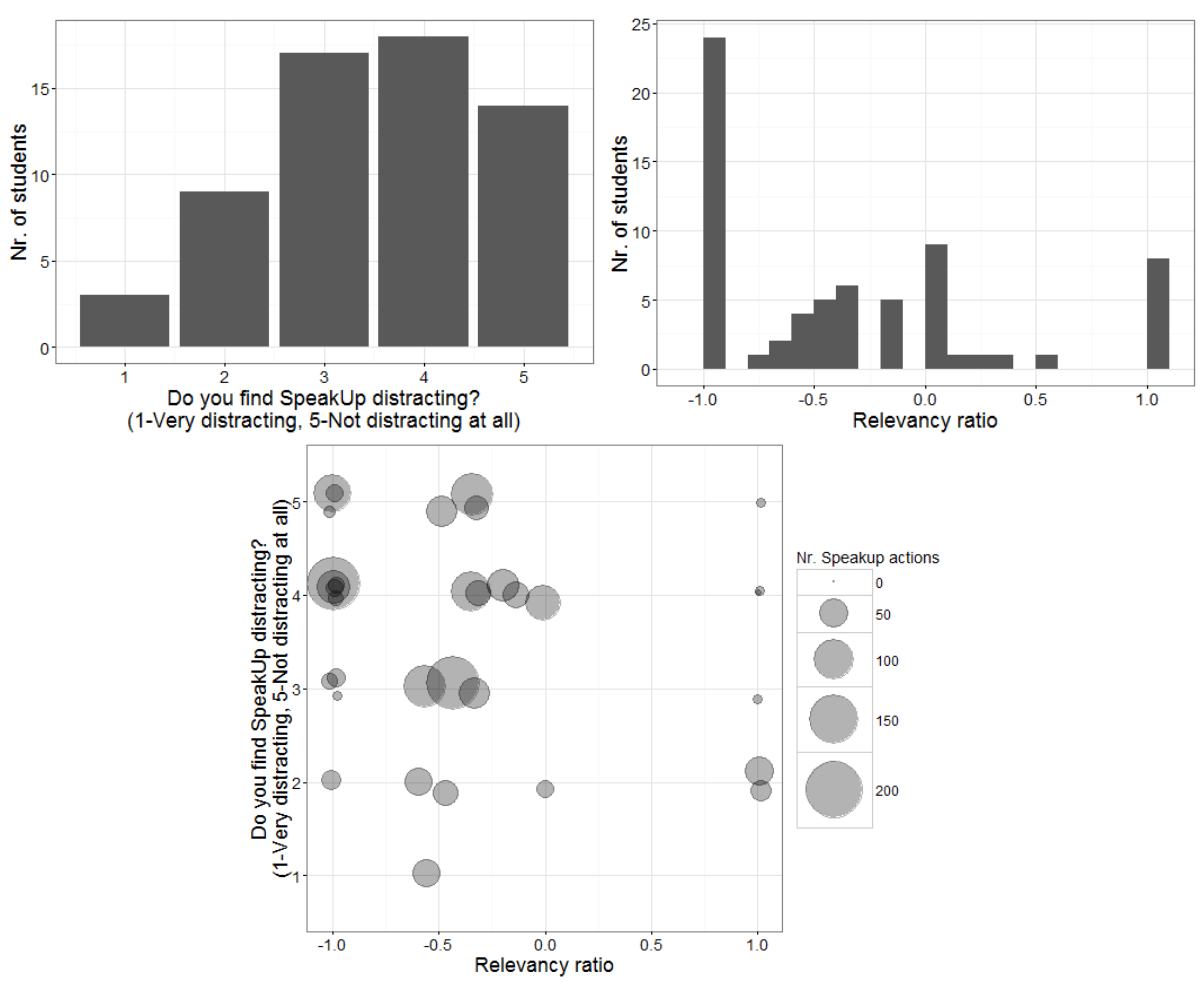

Fig. 5: Students' subjective opinions on whether SpeakUp is distracting them (top-left), and distribution of values of relevancy ratio of messages per student (top-right). At the bottom, both values are represented along each student's SpeakUp participation (size of the circles).

Figure 5 (bottom) puts both graphs in perspective and relates the students perception about SpeakUp and their behaviour using the app. Those students that considered the app less distracting ( 4 and 5 in the Likert-scale) where the ones who created more non-relevant messages. On the other hand, those that perceived the SpeakUp as more distracting, contributed with less messages but, in some cases, more relevant ones.

\subsection{Social interaction (Topic 3)}

The teachers [T_QUE] perceived SpeakUp as a mechanism that promoted interaction between them and students ( $\mu=5$ in a 5-point Likert scale) and among students themselves $(\mu=4.5)$. Regarding the interaction between teachers and students, the app helped teachers discover and handle important questions and comments. Among the drawbacks, the main concern was that SpeakUp messages required supervision, e.g., to avoid bullying and other interactions detrimental to the class dynamic.

In order to better understand how users interacted during the session, Figure 6 shows the amount of interaction registered in the face-to-face and SpeakUp channels. For face-to-face interaction, we have taken into account the amount of time spent in the communication (extracted from the video observation [R_VID]). For SpeakUp interactions we have counted the number of messages and votes generated by the users that 
were registered in the $\operatorname{logs}$ [T_COM, S_COM, SP_LOG]. Figure 6 reveals that the faceto-face channel supported mainly the interaction going from teachers to students, while SpeakUp concentrated most of the interactions between students.

The social network analysis shown in Figure 7 reveals that, far from existing multiple separate groups that interacted mostly among themselves (a common pattern in social networks), the network of interactions was rather dense. This may be caused by the fact of using anonymous users. Since it is not possible to know who sends the message, the user cannot decide to answer or follow just specific people, and mainly reacts to the content post by other users. As it is shown in Figure 7 (right), although 15 students were isolated, the interaction degree $(\mu=23.3, \tilde{x}=14.5, \sigma=23.6)$ is much higher than the number of students that could interact in a physical environment (e.g., 8 peers sitting around). Note that many of the students did not received any vote or comment (in-degree: $\mu=29.7, \tilde{x}=0, \sigma=60.9$ ), while, on the other hand, most of the students comment, answer or vote at least once (out-degree: $\mu=29.7, \tilde{x}=10.5, \sigma=45.5$ ).
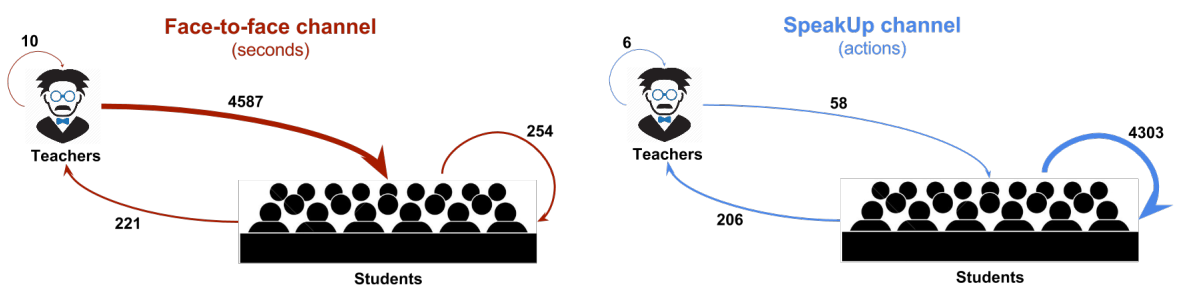

Fig. 6: Analyses of the communication direction in the face-to-face and SpeakUp channel.

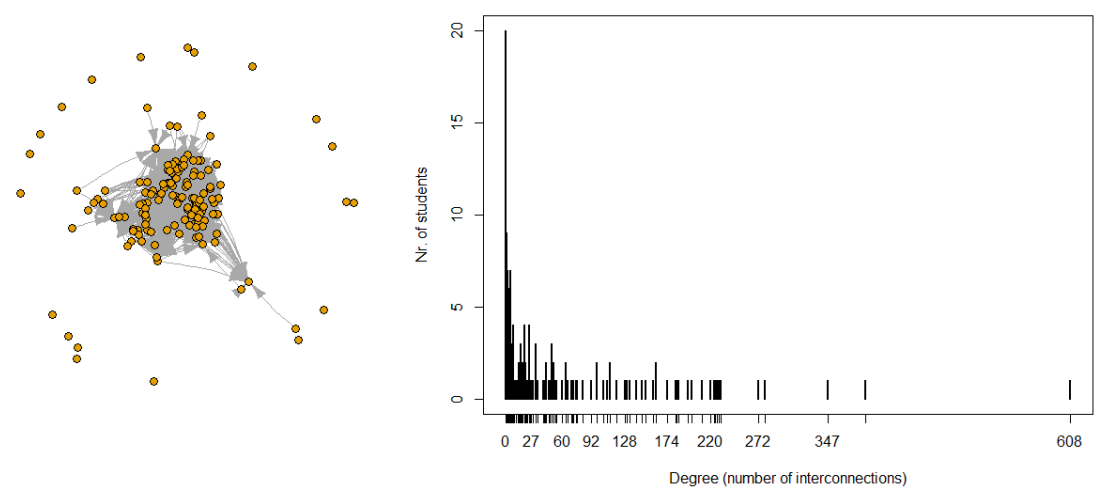

Fig. 7: Social network of SpeakUp interactions (left), and degree (number of interconnections) of the different SpeakUp users (right).

\subsection{Teaching style (Topic 4)}

As a general schema, the teachers of this course switch often during a same lecture to keep the course dynamic. Figure 8 shows which parts of the session were led by each 
one of the three teachers [R_VID] and the amount of relevant activity during such periods [T_COM, S_COM, SP_LOG]. Although, at first sight there was more activity in SpeakUp during the parts of the session led by Teacher1, it would be necessary to analyse more sessions in order to clarify if there is a dependence with the presentation style of the teacher (e.g., voice level, inflections, and physical language, duration), the support material (e.g., slides, videos, questionnaires, specific apps, ect.) or the specific content of the presentation. What seems to be more obvious is that high levels of relevant activity correspond to those moments when the teachers explicitly asked the students to use SpeakUp for specific learning purposes.

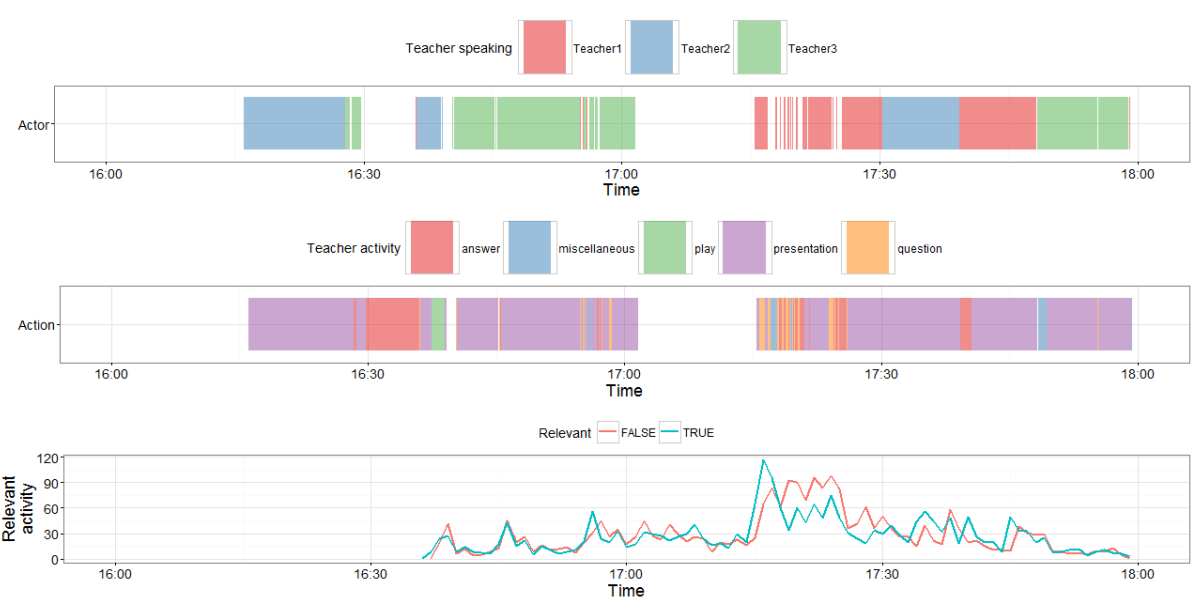

Fig. 8: Overview of actors, actions and resources used by the teachers during the session.

Regarding the way teachers used SpeakUp [T_QUE], before the session (see Figure 4), Teacher1 created the chatroom to be shared with the rest of the users. Then, during the session, while one teacher was presenting, the others checked SpeakUp to identify emerging questions or problems, vote (dis-like) non-relevant comments, and delete inappropriate ones. A significant difference between teachers styles [T_QUE, R_OBS, R_VID] is the way they interact with the tool. On the one hand, Teacher2 and Teacher3 did not use SpeakUp while they were lecturing. On the other hand, Teacher1 used it during his slots to satisfy his own teaching needs (e.g., he had a quick look to the messages when there was some noise, and checked in case of questions at the end of the presentation), and to support some learning activities (e.g., he asked students to answer some questions and give their opinions using the app).

As already mentioned in this section, the teachers found several benefits using SpeakUp that supported them in their practice. This tool provided them with awareness of a students back channel, and informed the interventions. However, they also pointed out that managing two simultaneous channels is demanding, specially difficult if teaching alone. Therefore, there is a need for finding an adequate scheme to handle face-to-face and computer-mediated interactions. 


\section{Discussion, conclusions and future work}

In our way towards understanding how to use social media effectively in the classroom, this paper analyses the use of SpeakUp in a face-to-face session with 3 teachers and 145 university students. In particular, we have explored to what extend SpeakUp favored situations that lead to learning, such as active participation (topic 1 - engagement), remaining on-task (topic 2 - attention), and social interaction (topic 3). Besides, we have explored the impact of the teaching style on the SpeakUp usage (topic 4).

The engagement results reveal that, even though the use of SpeakUp was optional, all students attending the session at least accessed the tool once. The clustering of users reveals that there is a gradient of involvement from passive to active users in terms of posting and voting. It should be noted that for most clusters there is usually a 2 to 1 ratio between the number of upvotes and the number of downvotes. Interestingly there is a cluster that we could dub the "SpeakUp police", who are the most active voters of all, and are mostly assigning negative votes in the opposite proportion.

Whereas many students wrote mainly non-relevant messages, compared to the 12 students who contributed mostly relevant messages, there was a significant amount of students who posted both relevant and not relevant messages, which means that using the tool for something else than learning is not just the activity of some bad apples. The results showing that the students with the lowest relevancy scores find the interaction in SpeakUp not distracting, whereas the students with the highest relevancy score find it the most distracting indicates a potential risk for the app usage if the high relevancy students start turning off their app.

One of the SpeakUp advantages highlighted by the teachers, and supported by the data analyses, refers to social interaction. First, students could not only share (doubts, problems, resources) but also comment and vote others contributions, favoring to get answers from peers without waiting for the teachers. Second, the app complemented the face-to-face channel. While most of the time teachers interacted orally with the students, the interaction between students was supported mostly via SpeakUp. Additionally, comparing the number of students reachable in the physical environment (e.g., 8 peers sitting around) versus the interaction degree in SpeakUp $(\mu=59.4, \tilde{x}=20)$, we may conclude that the tool contributed to increase the social network.

One of the aspects to be discussed is the twofold effect that anonymity might have on engagement, attention and interaction results. On the one hand, the anonymity could increase the usage of SpeakUp, since the students embrace the idea of not disclosing their identity (see Section 5.1). The flip side was that the anonymity brought more nonrelevant messages and required teachers to monitor the activity and intervene in case of inappropriate interaction (e.g. bullying).

Regarding the teacher impact on the student use of SpeakUp, it is noteworthy that when teachers asked the students to use the app in a certain way, the relevancy of the user activity increased significantly. Thus, the teacher role as scaffolding provider could contribute to a more effective use of the app.

Going back to the issue addressed in this case study, we can conclude that SpeakUp favored situations that led to learning, especially in terms of active participation (i.e., engagement) and social interaction. However, dealing with the attention, alternatives should be found in order to foster the appearance of relevant content (e.g., with teacher 
guidance). Nevertheless, the fact that the case study only covered the first session when the app was used by the students, could have introduce some additional distraction (novelty factor). Thus, it would be necessary to analyse the use of SpeakUp during the whole course, to see how student engagement, attention and interaction evolve. This study is currently under way, and is our most immediate avenue for future research.

\section{References}

1. Anderson, R.J., Anderson, R., VanDeGrift, T., Wolfman, S., Yasuhara, K.: Promoting interaction in large classes with computer-mediated feedback. In: Designing for change in networked learning environments, pp. 119-123. Springer (2003)

2. Bergstrom, T., Harris, A., Karahalios, K.: Encouraging initiative in the classroom with anonymous feedback. In: Campos, P., Graham, N., Jorge, J., Nunes, N., Palanque, P., Winckler, M. (eds.) Human-Computer Interaction - INTERACT 2011, Lecture Notes in Computer Science, vol. 6946, pp. 627-642. Springer Berlin Heidelberg (2011)

3. Blood, E., Neel, R.: Using student response systems in lecture-based instruction: Does it change student engagement and learning? Journal of Technology and Teacher Education 16(3), 375-383 (2008)

4. Creswell, J.W., Plano Clark, V.L., Gutmann, M.L., Hanson, W.E.: Advanced mixed methods research designs. In: Handbook of mixed methods in social and behavioral research, pp. 209-240. Sage Publications (2003)

5. Dewan, P.: An integrated approach to designing and evaluating collaborative applications and infrastructures. Computer Supported Cooperative Work (CSCW) 10(1), 75-111 (2001)

6. Dillenbourg, P.: What do you mean by collaborative learning. In: Collaborative-learning: Cognitive and computational approaches, vol. 1, pp. 1-15. Elsevier Publishing (1999)

7. Du, H., Rosson, M.B., Carroll, J.M.: Augmenting classroom participation through public digital backchannels. In: Proceedings of the 17th ACM International Conference on Supporting Group Work. pp. 155-164. GROUP '12, ACM, New York, NY, USA (2012)

8. Erickson, J., Siau, K.: E-ducation. CACM 46(9), 134-140 (2003)

9. Fies, C., Marshall, J.: Classroom response systems: A review of the literature. Journal of Science Education and Technology 15(1), 101-109 (2006)

10. Greene, J.C., Benjamin, L., Goodyear, L.: The merits of mixing methods in evaluation. Evaluation 7(1), 25-44 (2001)

11. Grotenbreg, G., Wong, S.B.J.: Using Pigeonhole $($ Live to elicit feedback, questions \& reinforce learning during lectures. CDLT Brief 16(2), 2-7 (2013)

12. Guba, E.G.: Criteria for assessing the trustworthiness of naturalistic inquiries. Journal of Theory, Research, and Development on Educational Communication and Technology 29(2), 75-91 (1981)

13. Harry, D., Green, J., Donath, J.: Backchan.nl: integrating backchannels with physical space. In: CHI'09. pp. 2751-2756. ACM (2008)

14. Holzer, A., Govaerts, S., Vozniuk, A., Kocher, B., Gillet, D.: Speakup in the classroom: Anonymous temporary social media for better interactions. In: Proceedings of the Extended Abstracts of the 32Nd Annual ACM Conference on Human Factors in Computing Systems. pp. 1171-1176. CHI EA '14, ACM, New York, NY, USA (2014)

15. Holzer, A., Govaerts, S., Vozniuk, A., Ondrus, J., Rigaud, D., Garbinato, B., Gillet, D.: Speakup - a mobile app facilitating audience interaction. In: ICWL'13, pp. 11-20. Springer (2013)

16. Horowitz, H.M.: Student response systems: Interactivity in a classroom environment. In: Proceedings of the Sixth Annual Conference on Interactive Instruction Delivery. Salt Lake City, UT (Febr 1988) 
17. Jensen, E.: Teaching with the brain in mind. Association for Supervision \& Curriculum Development (1998)

18. Johnson, R.B., Onwuegbuzie, A.J.: Mixed methods research: A research paradigm whose time has come. Educational Researcher 33(7), 14-26 (2004)

19. Jorrín-Abellán, I.M., Stake, R.E., Martínez-Monés, A.: The needlework in evaluating a CSCL system: the evaluand oriented responsive evaluation model. In: International Conference on Computer Supported Collaborative Learning. pp. 68-72. CSCL'09, International Society of the Learning Sciences, Rhodes, Greece (2009)

20. Kinzie, M.B., Whitaker, S.D., Hofer, M.J.: Instructional uses of instant messaging (IM) during classroom lectures. Educational Technology \& Society 8(2), 150-160 (2005)

21. Kreijns, K., Kirschner, P.A., Jochems, W.: Identifying the pitfalls for social interaction in computer-supported collaborative learning environments: a review of the research. Computers in human behavior 19(3), 335-353 (2003)

22. Kuznekoff, J.H., Munz, S., Titsworth, S.: Mobile Phones in the Classroom: Examining the Effects of Texting, Twitter, and Message Content on Student Learning. Communication Education 64(3), 344-365 (2015)

23. McCarthy, J.F., boyd, d.m.: Digital backchannels in shared physical spaces: Experiences at an academic conference. In: CHI '05 Extended Abstracts on Human Factors in Computing Systems. pp. 1641-1644. CHI EA '05, ACM, New York, NY, USA (2005)

24. Miles, M.B., Huberman, A.M.: Qualitative data analysis. An expanded sourcebook. SAGE Publications Inc. (1994)

25. Prince, M.: Does active learning work? a review of the research. Journal of engineering education 93(3), 223-231 (2004)

26. Reinhardt, W., Ebner, M., Beham, G., Costa, C.: How people are using twitter during conferences. In: Proc. of 5th EduMedia Conf. p. 145 (2009)

27. Retelny, D., Birnholtz, J.P., Hancock, J.T.: Tweeting for class: using social media to enable student co-construction of lectures. In: Poltrock, S.E., Simone, C., Grudin, J., Mark, G., Riedl, J. (eds.) CSCW (Companion). pp. 203-206. ACM (2012)

28. Sana, F., Weston, T., Cepeda, N.J.: Laptop multitasking hinders classroom learning for both users and nearby peers. Comput. Educ. 62, 24-31 (2013)

29. Scornavacca, E., Huff, S., Marshall, S.: Mobile phones in the classroom: if you can't beat them, join them. CACM 52(4), 142-146 (Apr 2009)

30. Seaman, J., Tinti-Kane, H.: Social media for teaching and learning. Tech. rep., Pearson Learning Solutions (2013)

31. Stake, R.: The art of case study research. Sage Publications, Thousand Oaks, CA (1995)

32. Stowell, J.R., Nelson, J.M.: Benefits of electronic audience response systems on student participation, learning, and emotion. Teaching of Psychology 34(4), 253-258 (2007)

33. Sung, Y.T., Chang, K.E., Liu, T.C.: The effects of integrating mobile devices with teaching and learning on students' learning performance: A meta-analysis and research synthesis. Computers \& Education 94, 252-275 (2016)

34. Trees, A.R., Jackson, M.H.: The learning environment in clicker classrooms: student processes of learning and involvement in large university-level courses using student response systems. Learning, Media and Technology 32(1), 21-40 (2007)

35. Worthington, D.L., Levasseur, D.G.: To provide or not to provide course powerpoint slides? the impact of instructor-provided slides upon student attendance and performance. Computers \& Education 85, 14-22 (2015)

36. Yardi, S.: The role of the backchannel in collaborative learning environments. In: Proceedings of the 7th International Conference on Learning Sciences. pp. 852-858. ICLS '06, International Society of the Learning Sciences (2006) 